${ }^{1} \mathrm{H}$ D Mitchell, 2,3D A Lewis, 'K Marsh, ' $\mathbf{G}$ Hughes. 'Health Protection Agency, London, UK; ${ }^{2}$ National Institute for Communicable Diseases, Johannesburg, South Africa; ${ }^{3}$ University of Witwatersrand, Johannesburg, South Africa

Background Little is known about the recent epidemiology and public health impact of Trichomonas vaginalis infection in England. We investigated the distribution and risk factors of this common sexually transmitted infection (STI) and assessed whether the potential burden of infection could warrant a review of existing screening guidelines.

Methods We used data from the Genitourinary Medicine Clinic Activity Dataset (GUMCAD) over a three year period (2009-2011) to investigate the characteristics of patients diagnosed with $T$. vaginalis, and to describe the distribution of cases in England. Case records were linked within each GUM clinic using the local patient identifier. Socio-demographic and clinical risk factors associated with a diagnosis of $T$. vaginalis were explored using multivariable logistic regression.

Results Rates of $T$. vaginalis infection were highest in London and the West Midlands. Risk of a T. vaginalis diagnosis was strongly associated with older age in comparison to those aged 20-24 years, non-white ethnicity, in particular black Caribbean (adjusted Odds Ratio $[\mathrm{aOR}]=4.23,95 \%$ CI 3.98-4.50 in women; $\mathrm{aOR}=8.00,95 \%$ CI 6.48-9.87 in men) and black 'other' ( $\mathrm{aOR}=4.13,95 \%$ CI 3.80 4.49 in women; $\mathrm{aOR}=5.75,95 \% \mathrm{CI} 4.22-7.83$ in men) ethnic groups and birth in the Caribbean $(\mathrm{aOR}=1.27,95 \%$ CI $1.16-1.38$ in women; $\mathrm{aOR}=1.63,95 \%$ CI $1.28-2.09$ in men) compared to the UK. Current gonorrhoea ( $\mathrm{aOR}=3.66,95 \%$ CI 3.30-4.05) or chlamydia (aOR $=1.58,95 \%$ CI 1.49-1.68) infection was an important risk factor for a diagnosis of $T$. vaginalis in women.

Conclusion This study has characterised important patient groups at risk of $T$. vaginalis infection and allowed identification of areas of higher prevalence. Our results suggest that further research is needed to identify the public health benefits and feasibility of changing clinic screening protocols among at risk groups in these areas.

\section{P3.092 PREVALENCE OF TRICHOMONAS VAGINALIS IN FRANCE AND COMPARISON WITH CHLAMYDIA TRACHOMATIS AND NEISSERIA GONORRHOEAE AS DETERMINED BY THE APTIMA TRICHOMONAS VAGINALIS NUCLEIC ACID AMPLIFICATION ASSAY}

doi:10.1136/sextrans-2013-051184.0551

M Dautigny, A Ebel, S Merlin, A Souchet, C Ronsin. Laboratoire Biomnis, Ivry sur seine cedex, France

In France, few data on prevalence of Trichomonas vaginalis in the general population as well as in high-risk populations exist. We determined Trichomonas vaginalis (TV) together with Chlamydia trachomatis (CT) and Neisseria gonorrhoeae (NG) prevalence, in men and women with or without symptoms of sexually transmitted infection (STI), undergoing CT and NG screening in France.

683 men and 2432 women were enrolled on a basis of either urethral, cervical or vaginal swabs, urine or sperm samples, using the APTIMA Trichomonas vaginalis assay (ATV, Hologic|Gen-Probe) and the APTIMA Combo 2 assay for CT and NG (AC2, Hologic|GenProbe), respectively.

Overall TV, CT and NG prevalence in women was $0.7 \%, 6.13 \%$ and $0.49 \%$, respectively, and in men $0.44 \%, 9.52 \%$ and $3.07 \%$, respectively. All in all 20 patients were positive for TV, 17 women and 3 men, with the highest TV prevalence in women of $>40$ years (1.59\%), while CT (11.58\%) and NG $(0.87 \%)$ prevalence was highest in women $<30$ years.

Co-infections were relatively low: No subject was coinfected with $\mathrm{CT} / \mathrm{TV}$, most probably due to prevalence in different age groups, and 5 subjects were coinfected with CT/NG, 3 women and 2 men.
The CT prevalence reported here is higher than what was reported in earlier epidemiologic studies in France. The low prevalence of TV may be due to a bias in tested population.

In this study we determined prevalence of the 3 infections in women and men in all age groups and in relation to the specimen type. Furthermore, we provide a detailed analysis of the results obtained from the 20 TV positive cases. Further studies are needed to estimate the prevalence of TV in other targeted populations.

\section{P3.093 SIMILAR, LOW PREVALENCE OF TRICHOMONAS VAGINALIS IN THREE PATIENT COHORTS FROM GENERAL PRACTITIONERS, A POPULATION BASED CHLAMYDIA SCREENING STUDY AND AN STI-CLINIC}

doi:10.1136/sextrans-2013-051184.0552

'T H Geelen, 1,2 C J P A Hoebe, 1,2A Dirks, 3J E van Bergen, 1,2 $\mathrm{N} \mathrm{H} \mathrm{T} \mathrm{M} \mathrm{Dukers-Muijrers,}$ 'P F G Wolffs. 'Maastricht University Medical Center, Maastricht, The Netherlands, ${ }^{2}$ Department of Sexual Health, Infectious Diseases and Environment, Public Health Service, South Limburg, Geleen, The Netherlands; ${ }^{3}$ STI Aids The Netherlands, Amsterdam, The Netherlands

Background Among sexually transmitted infections (STIs), Trichomonas vaginalis (TV) is the most common non-viral STI worldwide. However, in Europe, a limited number of studies, mostly on specific risk groups, have described a low TV prevalence. Therefore a large multi-cohort study was performed to investigate the prevalence of TV among three distinct Dutch patient populations. Additionally, the relationship between Chlamydia trachomatis (CT) and TV coinfection was assessed.

Methods 2089 participants, men and women, were included between 2008 and 2012 from three distinct cohorts. A total of 575 participants from the population-based national Chlamydia Screening Intervention (CSI) study, 465 attendees of the STI-clinic South Limburg and 602 patients from general practitioners (GPs) were included. An additional number of CT positives $(n=447)$ was included to assess TV and CT co-infection. All participants were assessed for TV using real-time PCR.

Results The overall prevalence of TV was $0.9 \%$ among the three distinct cohorts and no significant differences between the 3 study populations were observed (GP patients $(1.5 \%)$, STI clinic $(0.6 \%)$ and population-based cohort $(0.7 \%))$. TV was found in $0.7 \%$ of the CT positives and a similar $1.1 \%$ among CT negatives. In contrast to CT prevalence which significantly decreased with increasing age, TV prevalence was highest in the age group $>40$ years $(2 \%)$, but did not differ significantly from other age-groups.

Conclusion This large multi-cohort study confirms the low burden for TV in the Netherlands $(<2 \%)$ as in most European countries. We have no indication for standard TV testing in regular care.

\section{P3.094 TRICHOMONAS VAGINALIS INFECTION AMONG WOMEN OF REPRODUCTIVE AGE GROUP IN A COSMOPOLITAN SETUP}

doi:10.1136/sextrans-2013-051184.0553

A R Almadi. Cyberjaya University College of Medical Sciences, Cyberjaya, Malaysia

Trichomoniasis is one of the most common sexually transmitted infections in humans. In a prospective, observational study, 380 women attending the family planning (FP) clinic and Sexually Transmitted Diseases (STD) clinic in Kuala Lumpur were enrolled. Patient's information was obtained from the standardised medical records and voluntarily completed questionnaires. Three vaginal swabs from posterior fornix were taken from each patient. Different staining methods and cultivation in Diamonds medium were performed for the collected samples. Study subjects recruited in this survey were mostly young, with a geometric mean of 37.31 years (FP clinic) and 32.06 years (STD clinic). Malay, Chinese, Indians and others ethnic 